\title{
GMR
}

\section{Prevalence of human papillomavirus genotypes among women with cervical lesions in the Shaanxi Province of China}

\author{
J. Li', Y.Y. Wang', X. Nan', X.F. Tian², T. Yan², P. Wang' ${ }^{2}$, Y. Yin', Y. Liu', R. Yuan', \\ G.Q. Wang ${ }^{2}$ and Y.L. Fu ${ }^{2}$ \\ ${ }^{1}$ Basic Research Center, Shaanxi Provincial Tumor Hospital, \\ Xi'an Jiaotong University, Xi'an, China \\ ${ }^{2}$ Gynecologic Oncology Center, Shaanxi Provincial Tumor Hospital, \\ Xi'an Jiaotong University, Xi'an, China \\ Corresponding author: J. Li \\ E-mail: wonder_689@163.com \\ Genet. Mol. Res. 15 (1): gmr.15017181 \\ Received July 10, 2015 \\ Accepted September 28, 2015 \\ Published March 31, 2016 \\ DOI http://dx.doi.org/10.4238/gmr.15017181
}

\begin{abstract}
This study aimed to investigate human papilloma virus (HPV) genotypes among women with cervical lesions in Shaanxi Province, China, to obtain information regarding cervical lesion prevention and treatment. The study included 4508 HPV-positive subjects; cervical swab specimens were collected and tested for HPV infection status and HPV genotypes using polymerase chain reaction and reverse dot-blot hybridization. Women positive for HPV with cervical lesions, including chronic cervicitis, cervical intraepithelial neoplasia, and cervical squamous cell carcinoma (SCC), were examined; HPV-positive women with no cervical lesions were controls. Data were pooled and weighted estimates have been presented. For women with no cervical lesions and positive for one HPV genotype, HPV 52, 16, 58, 81, 33, and 56 were the most common; for multiple-HPV genotype infection, HPV 16, 52, 6, 18, 58, and 66 were the most common. Collectively, HPV 16, 58, 52, 18, 33, and 81 were the most common in women with cervical lesions. HPV 16 comprised $26.71 \%$
\end{abstract}


of single-genotype and $15.64 \%$ of multiple-genotype infections. The proportion of HPV-16-positive cases was $29.15 \%$, which was the highest among all HPV genotypes $(P<0.01)$. Single-HPV genotype infection was the most common in cervical HPV infection $(77.48 \%)$; infection with two HPV genotypes comprised $72.22 \%$ of multiple-genotype infections. The proportion of single-low-risk HPV genotype infections decreased with increase in cervical lesion severity; there were no single- or multiple-lowrisk genotype HPV infections in cervical SCC patients. The proportion of multiple-genotype HPV infections with at least one high-risk genotype increased with cervical lesion severity.

Key words: HPV; Genotype; Chronic cervicitis;

Cervical intraepithelial neoplasia; Cervical squamous cell carcinoma

\section{INTRODUCTION}

Human papillomavirus (HPV) is a double-stranded DNA virus with a genome of approximately $8 \mathrm{~kb}$. HPV can infect epithelial tissues through micro-abrasions or other epithelial trauma and replicate in the basal cells of the stratified epithelium. HPV infection is usually subclinical, and persistent infection can lead to tissue damage, malignancy (Lowy and Schiller, 2006), and, ultimately, cervical cancer (Cuschieri et al., 2004). HPV infection is rather common in China and it is estimated that $70-80 \%$ of women will be infected at least once in their lifetime and $10-15 \%$ of the cases will turn into persistent infections (Steben and Duarte-Franco, 2007). With 130,000 newly diagnosed cervical cancer cases every year, which accounts for almost $50 \%$ of new cases in the world, China has become a country with a high morbidity rate for cervical cancer (Steben and Duarte-Franco, 2007). HPV has multiple genotypes and the prevalence of these genotypes is associated with distinct populations and regionality (de Sanjosé et al., 2007). Our study investigates the prevalence of HPV genotypes among women in the Shaanxi Province in China using polymerase chain reaction (PCR) and reverse dot-blot hybridization assay. Our results could provide valuable information regarding the prevention and treatment of cervical cancer.

\section{MATERIAL AND METHODS}

\section{Study population and enrollment}

Cervical specimens were collected from patients enrolled in the Shaanxi Province Breast Cancer and Cervical Cancer Screening Program, Shaanxi Province Opportunistic Screening Program, and the Shaanxi Provincial Tumor Hospital. Additional samples were obtained from physical examination specimens from various populations in Shaanxi. Selection criteria were as follows: permanent resident in Shaanxi Province; sexually active for at least a year; no history of cervical colonization or hysterectomy; no systemic infection or autoimmune diseases; no pelvic examination, intravaginal drug administration, or sexual activity 3 days before the examination; psychologically sound; and not pregnant. All specimens were tested for HPV genotypes; due to the nature of our study, HPV-negative subjects were not included. A total of 3987 women with cervical lesions were enrolled (age range, 19-69 years; median age, 43.96 years); 521 women positive for 
HPV with no cervical lesions were enrolled as the control group (NC) (age range, 21-63 years; median age, 43.87 years). In addition, the study included 697 chronic cervicitis patients (age, 1961 years; median age, 41.26 years). The cervical intraepithelial neoplasia (CIN) group consisted of 1543 cases $(\mathrm{CIN} \mathrm{I}=528, \mathrm{CIN} \mathrm{II} \mathrm{=601,} \mathrm{CIN} \mathrm{III} \mathrm{=414),} \mathrm{with} \mathrm{an} \mathrm{age} \mathrm{range} \mathrm{of} \mathrm{24-62} \mathrm{years} \mathrm{and} \mathrm{a}$ median age of 43.33 years. The squamous cell carcinoma (SCC) group included 1747 cases (SCC stage I = 339, SCC stage II = 934, SCC stage III = 379, SCC stage IV =95), with an age range of 26-69 years and a median age of 45.67 years. Cases of cervical adenocarcinoma, adenosquamous carcinoma, and undifferentiated carcinoma were excluded from this study. All diagnoses were confirmed by a pathologist. Within the SCC group, there were 409 well-differentiated tumors, 885 moderately differentiated tumors, and 453 poorly differentiated tumors. There were 1063 cases with no lymph node involvement and 684 cases with lymph node metastasis present.

\section{Equipment and reagents}

HPV genotype detection reagents were purchased from Ya Neng Biotechnology Company (Shenzhen, China). This reagent can detect 18 high-risk HPV genotypes (16, 18, 31, 33, 35, 39, 45, 51, 52, 53, 56, 58, 59, 66, 68, 73, 82, 83) and 5 low-risk HPV genotypes (6, 11, 42, 43, 81). The Applied Biosystems $^{\circledR} 7500$ Real-Time PCR System (USA) was used for PCR experiments. The bio-safety cabinet (HFsafe-1200TE) was provided by Li Kang Science and Technology Company (Shanghai, China). The ultra-low temperature freezer (DW-86L386) was purchased from Haier Company (China). The hybridization oven (FYY-3) was provided by Xing Hua Company (Ya Neng Biotechnology Company). Nylon membranes (pore size $=0.45 \mu \mathrm{M}$, thickness $6.0 \pm 0.5 \mathrm{~mm}$ ) were purchased from Pall (USA).

\section{Specimen collection and preservation}

A speculum was inserted into the subject's vagina, which helped spread the vagina open and allowed access to the cervix. Vaginal secretions were swabbed from the cervix and then a sample was collected from the outer opening of the cervix by scraping with an endocervical brush. The brush was rotated 4-5 times in the central opening of the cervix to obtain sufficient cells. The brush was then broken off and the tip of the brush was placed into a tube filled with preservative liquid. The tube was sealed, labeled, and stored at $-80^{\circ} \mathrm{C}$ until further testing. DNA extraction (DNA extraction kit, Bioteck, Beijing), PCR amplification (PCR kit, Bioteck), hybridization, incubation, and color development were all performed according to the manufacturer instructions (Li et al., 2013). For analysis, blue dots were considered positive and HPV genotype analysis was done on a readout system. Experiments were performed with positive controls (genotyping sample) to ensure the quality of analysis.

\section{Statistical analysis}

Data were collected and analyzed with the SPSS 16.0 software (SPSS, Chicago, IL, USA). The Student $t$-test and the $\chi^{2}$ test were used, and $\mathrm{P}<0.05$ was considered significant.

\section{RESULTS}

Table 1 shows the prevalence of single-genotype HPV infections in women with cervical lesions. The most common genotypes were HPV 52, 16, 58, 81, 33, and 56 for women with no 
cervical lesions (NC group); HPV 16, 52, 81, 58, 6, and 43 for the chronic cervicitis group; HPV 16, 52, 58, 33, 18, and 31 for the CIN I group; HPV 16, 52, 58, 33, 18, and 56 for the CIN II group; HPV 16, 58, 52, 18, 33, and 68 for the CIN III group; HPV 16, 58, 52, 18, 33, and 56 for the SCC stage I group; HPV 16, 58, 18, 52, 33, and 56 for the SCC stage II group; HPV 16, 58, 18, 52, 33, and 68 for the SCC stage III group; and HPV 16, 58, 18, 52, 33, and 52 for the SCC stage IV group. The most prevalent genotypes in single-genotype infection or single-high-risk genotype infection were HPV 16, 58, 52, 18, 33, and 56, and in single-low-risk genotype infections were HPV 81, 6, 43,42 , and 11.

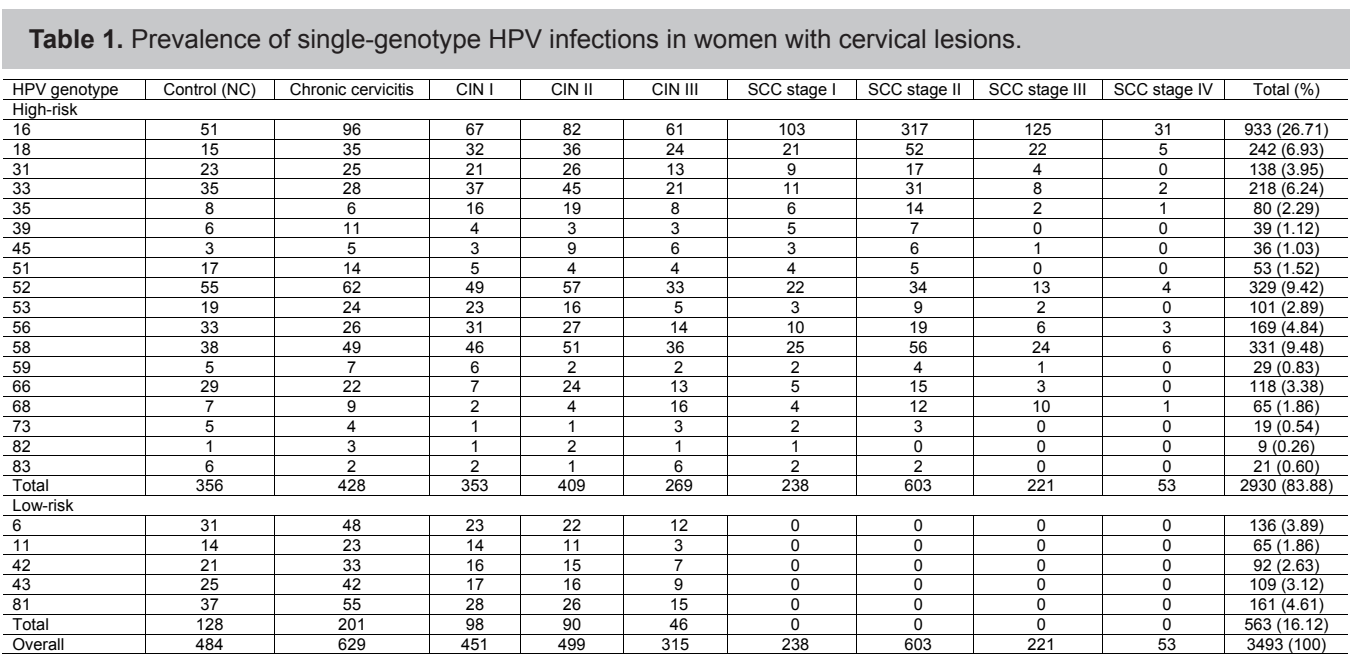

$\mathrm{NC}=$ no cervical lesions; $\mathrm{CIN}=$ cervical intraepithelial neoplasia; $\mathrm{SCC}=$ squamous cell carcinoma.

Table 2 shows the prevalence of multiple-genotype HPV infections in women with cervical lesions. The most common genotypes were HPV 16, 52, 6, 18, 58, and 66 for the NC group; HPV $16,52,58,18,56$, and 6 for the chronic cervicitis group; HPV 16, 58, 18, 56, 6, and 52 for the CIN I group; HPV 16, 58, 52, 81, 18, and 6 for the CIN II group; HPV 16, 52, 81, 6, 58, and 18 for the CIN III group; HPV 16, 52, 18, 58, 6, and 81 for the SCC stage I group; HPV 16, 52, 58, 18, 56, and 81 for the SCC stage II group; HPV 16, 81, 52, 58, 42, and 18 for the SCC stage III group; and HPV 16, $52,81,18,58$, and 42 for the SCC stage IV group. The most prevalent genotypes were HPV 16, 52 , $58,81,18$, and 6 in multiple-genotype infections; HPV 16, 52, 58, 18, 56, and 33 in multiple-highrisk genotype infections; and HPV 81, 6, 42, 43, and 11 in multiple-low-risk genotype infections.

Table 3 shows the prevalence of single- or multiple-genotype HPV infections in women with cervical lesions. The proportion of multiple-genotype HPV infections was only $7.10 \%$ in the NC group, and no more than two genotypes were detected in one specimen. In the chronic cervicitis group, the proportion of patients with two genotypes was $9.88 \%$, and at most 3 genotypes were detected in one specimen. In the CIN I, II, and III groups, the proportion of multiple-genotype HPV infections was $14.70,16.98$, and $23.91 \%$, respectively. A maximum of 4 , 5, or 6 genotypes were identified in one specimen in the CIN I, II or III groups, respectively. In the SCC stage I, II, III and IV groups, the proportion of multiple-genotype HPV infections was 29.79, 35.44, 41.69, and 44.21\%, respectively. A maximum of $6,7,7$ or 5 genotypes were identified in one specimen in the SCC stage I, II, III, or IV groups, respectively. The proportion of multiple-genotype HPV infections was 
$18.02 \%$ in the CIN group (I, II, and III combined) and 36.18\% in the SCC group (stages I, II, III, and IV combined). The proportion of multiple-genotype HPV infections in chronic cervicitis was not significantly different from that in the NC group $(P>0.05)$. The difference between the proportion of multiple-genotype HPV infections in SCC stage III and SCC stage IV was not significant (P > $0.05)$. However, this difference was significant between the chronic cervicitis or SCC group and the CIN group $(P<0.01)$.

Table 2. Prevalence of multiple-genotype HPV infections in women with cervical lesions (each subtype in the multiple infections was counted separately).

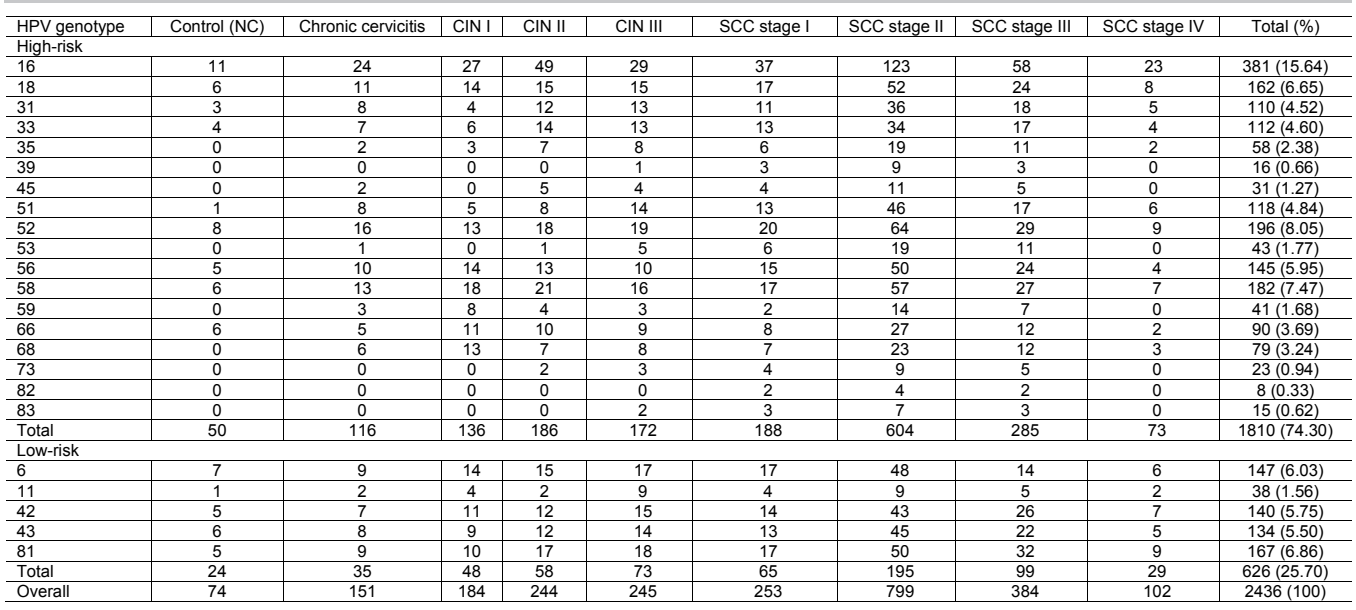

$\mathrm{NC}=$ no cervical lesions; $\mathrm{CIN}=$ cervical intraepithelial neoplasia; $\mathrm{SCC}=$ squamous cell carcinoma.

Table 3. Distribution of single- and multiple-genotype HPV infections in women with cervical lesions.

\begin{tabular}{l|c|c|c|c|c|c|c|c}
\hline \multirow{2}{*}{ Group } & \multirow{2}{*}{$\mathrm{N}$} & \multicolumn{9}{c}{ HPV infection (\%) } \\
\cline { 3 - 9 } & & $\begin{array}{c}\text { Single } \\
\text { genotype }\end{array}$ & $\begin{array}{c}\text { Two } \\
\text { genotypes }\end{array}$ & $\begin{array}{c}\text { Three } \\
\text { genotypes }\end{array}$ & $\begin{array}{c}\text { Four } \\
\text { genotypes }\end{array}$ & $\begin{array}{c}\text { Five } \\
\text { genotypes }\end{array}$ & $\begin{array}{c}\text { Six } \\
\text { genotypes }\end{array}$ & $\begin{array}{c}\text { Seven } \\
\text { genotypes }\end{array}$ \\
\hline NC & 521 & $484(92.90)$ & $37(7.10)$ & 0 & 0 & 0 & 0 & 0 \\
\hline Chronic cervicitis & 697 & $629(90.24)$ & $53(7.60)$ & $15(2.15)$ & 0 & 0 & 0 & 0 \\
\hline CIN I & 528 & $451(85.42)$ & $54(10.23)$ & $16(3.03)$ & $7(1.33)$ & 0 & 0 & 0 \\
\hline CIN II & 601 & $499(83.03)$ & $75(12.48)$ & $17(2.83)$ & $6(1.00)$ & $4(0.67)$ & 0 & 0 \\
\hline CIN III & 414 & $315(76.09)$ & $66(15.94)$ & $24(5.80)$ & $5(1.21)$ & $3(0.72)$ & $1(0.24)$ & 0 \\
\hline SCC stage I & 339 & $238(70.21)$ & $68(20.06)$ & $22(6.49)$ & $6(1.77)$ & $4(1.18)$ & $1(0.29)$ & 0 \\
\hline SCC stage II & 934 & $603(64.56)$ & $236(25.27)$ & $67(7.17)$ & $18(1.93)$ & $7(0.75)$ & $2(0.21)$ & $1(0.11)$ \\
\hline SCC stage III & 379 & $221(58.31)$ & $115(30.34)$ & $29(7.65)$ & $7(1.85)$ & $4(1.06)$ & $2(0.53)$ & $1(0.26)$ \\
\hline SCC stage IV & 95 & $53(55.79)$ & $29(30.53)$ & $9(9.47)$ & $3(3.16)$ & $1(1.05)$ & 0 & 0 \\
\hline Total & 4508 & $3493(77.48)$ & $733(16.26)$ & $199(4.41)$ & $52(1.15)$ & $23(0.51)$ & $6(0.13)$ & $2(0.04)$ \\
\hline
\end{tabular}

$\mathrm{NC}=$ no cervical lesions; $\mathrm{CIN}=$ cervical intraepithelial neoplasia; SCC $=$ squamous cell carcinoma.

Table 4 showed the distribution of high- or low-risk genotype HPV infections among women with cervical lesions. The proportion of single-low-risk genotype HPV infections in the chronic cervicitis group $(28.84 \%)$ was significantly higher than in any other group $(P<0.05)$. Single-highrisk genotype HPV infections were very common in all groups, but the proportion decreased with the stage of SCC $(P<0.05)$. The proportion of multiple-low-risk genotype HPV infections was low in all groups, with none occurring in the SCC group. However, multiple-high-risk genotype HPV infections increased with the severity of cervical lesions. The proportion of high-risk HPV infections was $1.92 \%$ in the NC group compared with $24.21 \%$ in the SCC stage IV group. The difference 
between the chronic cervicitis group and the NC group was not significant $(P>0.05)$ in terms of high-risk HPV infections, while this difference was significant between the chronic cervicitis group and the CIN group. The proportion of multiple-high-risk genotype HPV infections was significantly lower in the NC group than in the CIN or SCC groups $(P<0.01)$. The data for multiple-highand low-risk genotype HPV infections showed the same trend as multiple-high-risk genotype HPV infections.

Table 4. Distribution of high- or low-risk-genotype HPV infections among women with cervical lesions.

\begin{tabular}{l|c|c|c|c|c|c}
\hline \multirow{2}{*}{ Group } & \multirow{2}{*}{$\mathrm{N}$} & \multicolumn{5}{|c}{ HPV infection (\%) } \\
\cline { 3 - 7 } & & Single-low-risk & Single-high-risk & Multiple-low-risk & Multiple-high-risk & Multiple-high and low-risk \\
\hline NC & 521 & $128(24.57)$ & $356(68.33)$ & $18(3.45)$ & $10(1.92)$ & $9(1.73)$ \\
\hline Chronic cervicitis & 697 & $201(28.84)$ & $428(61.41)$ & $34(4.88)$ & $19(2.73)$ & $15(2.15)$ \\
\hline CIN I & 528 & $98(18.56)$ & $353(66.86)$ & $16(3.03)$ & $39(7.39)$ & $22(4.17)$ \\
\hline CIN II & 601 & $90(14.98)$ & $409(68.05)$ & $15(2.49)$ & $45(7.49)$ & $42(6.99)$ \\
\hline CIN III & 414 & $46(11.11)$ & $269(64.98)$ & $3(0.72)$ & $53(12.80)$ & $43(10.39)$ \\
\hline SCC stage I & 339 & 0 & $238(70.21)$ & 0 & $54(15.93)$ & $47(13.86)$ \\
\hline SCC stage II & 934 & 0 & $603(64.56)$ & 0 & $168(17.99)$ & $163(17.45)$ \\
\hline SCC stage III & 379 & 0 & $221(58.31)$ & 0 & $80(21.11)$ & $78(20.58)$ \\
\hline
\end{tabular}

$\mathrm{NC}=$ no cervical lesions; $\mathrm{CIN}=$ cervical intraepithelial neoplasia; $\mathrm{SCC}=$ squamous cell carcinoma .

\section{DISCUSSION}

HPV infection is one of the confirmed pathogenic factors in cervical cancer (Tovar et al., 2008). HPV 16 and 18 can be found in most cervical cancers (Stanley et al., 2007) and more than $50 \%$ of cervical cancer patients are HPV 16 positive (Grce et al., 2010). However, the prevalence of HPV infections is distinctly different between countries, ethnicities, and even regions within the same country (Clifford et al., 2005). It has been widely reported that most HPV infections are cleared within 12 months and only a small number become persistent infections. High-risk genotype HPV infections are usually difficult to clear and can increase the risk of CIN III by 100- to 300-fold (Bekkers et al., 2004).

Regional disparities in HPV genotypes have come into focus as the etiology of cervical cancer develops because the prevalence of a particular HPV genotype could provide more information on the carcinogenicity and epidemiology of the virus in one specific region. Genotype screening on a large scale could detect the prevalence of HPV genotypes (single or multiple genotype; high-risk, low-risk or both) in infected people who are asymptomatic (Tovar et al., 2008; $\mathrm{Li}$ et al., 2014) and the genotypes prevalent in women with cervical lesions. There are already several reports on this subject in China (Li et al., 2009; Muhanmode and Liu, 2010; Yang et al., 2010) but the numbers of enrolled participants were small.

Our study analyzed specimens from HPV-positive women with or without cervical lesions ( $N=521$ or 3987 , respectively). Among women positive for a single genotype, the most common genotypes were HPV 52, 16, 58, 81, 33, and 56 for women with no cervical lesions (NC group); HPV 16, 52, 81, 58, 6, and 43 for the chronic cervicitis group; HPV 16, 52, 58, 33, 18, and 56 for the CIN group; and HPV 16, 58, 18, 52, 33, and 56 for the cervical SCC group. Our results show that the most prevalent genotypes for single-genotype infections were distinct in different groups. HPV 16 was the most common genotype for single-genotype infections in all groups except the NC group, and this finding is consistent with previous reports (Clifford et al., 2005; Sandri et al., 2009; Li et al., 2011). Other common genotypes were first identified in our previous studies (Sandri et al., 2009; Muhanmode and Liu, 2010; Yang et al., 2010; Li et al., 2009, 2011; Rao et al., 2012). 
The most prevalent genotypes in single-genotype or single-high-risk genotype HPV infections were HPV 16, 58, 52, 18, 33, and 56, while in single-low-risk genotype HPV infections the most prevalent genotypes were $81,6,43,42$, and 11 .

Among patients with multiple-genotype HPV infections, the most common genotypes were HPV 16, 52, 6, 18, 58, and 66 for women with no cervical lesions (NC group); HPV 16, 52, 58, 18 , 56 , and 6 for the chronic cervicitis group; HPV 16, 58, 52, 6, 81, and 18 for the CIN group; and HPV $16,52,58,81,18$, and 6 for the cervical SCC group. Collectively, the most common HPV genotype in women with cervical lesions was HPV 16, which accounted for $37.54 \%(381 / 1015)$ of multiplegenotype HPV infections. The second and third most common high-risk genotypes were HPV 52 and 58 , accounting for $19.31 \%$ (196/1015) and $17.93 \%$ (182/1015) of multiple-genotype infections, respectively (Zhang et al., 2009). HPV 52 and 58 are prevalent in Asia and play important roles in multiple-genotype infections (Trottier et al., 2006; Wu et al., 2006).

The most common genotypes overall were HPV $16,52,58,18,33$, and 81 , accounting for $75.73 \%$ (3414/4508) of HPV infections in all patients. This shows that the prevalence of HPV in Shaanxi Province is due to multiple-genotype infections. While it has been suggested that multipleHPV genotype infections do not correlate with the severity of cervical lesions (Sandri et al., 2009), other studies have shown a positive correlation between the two factors (Tao et al., 2006). The proportion of single- and multiple-genotype infections was 92.90 and $7.10 \%$ in the NC group, 90.24 and $9.76 \%$ in the chronic cervicitis group, 81.98 and $18.12 \%$ in the CIN group, and 63.82 and $36.18 \%$ in the SCC group, respectively. Therefore, the proportion of multiple-genotype infections increased with the severity of cervical lesions while the proportion of single-genotype infections decreased $(P<0.01)$. We identified numerous genotypes in multiple-genotype HPV infections in our study, many of which differed from previous reports (Zhao and Zhang, 2009). Overall, the proportion of single- and multiple-genotype infections was 77.48 and $22.52 \%$, respectively. These values are $20 \%$ higher than the findings reported by Bhatla et al. (2008) and $43 \%$ lower than the findings from Sandri et al. (2009). The overall incidence of double-genotype infections was $16.26 \%$ and the incidence of three or more genotype infections was $6.26 \%$. The single-genotype infections were more common in HPV infection-related cervical lesions $(P<0.01)$. Moreover, there were fewer multiple-genotype infections with more than three genotypes in women with cervical lesions, contrary to previous reports ( $\mathrm{Li}$ et al., 2012). Double-genotype infections accounted for $72.22 \%$ of all multiple infections and were the most common scenario in multiple infections.

Spinillo et al. (2009) found that multiple-genotype HPV infections could lead to the progression of cervical lesions. The risk of disease progression tripled with multiple-high-risk genotype infections compared to single-high-risk genotype infections. Our study found that the proportion of singlelow-risk genotype HPV infections decreased with the severity of cervical lesions $(P<0.01)$ but the proportion of multiple-genotype infections with at least one high-risk genotype increased with the severity of cervical lesions $(P<0.01)$. These results were consistent with previous studies (Tao et al., 2006). Single-high-risk genotype infections were common in all groups, but the proportion of single-high-risk infections decreased with the advancement of SCC stage. These findings suggest that multiple-genotype HPV infections play a role in precancerous lesions and cervical cancer progression. We hypothesize that the immune system is unable to clear the HPV infection efficiently when dealing with multiple-HPV genotypes. However, further investigation is required to determine if different genotypes act synergistically during disease progression.

We also found that single- or multiple-low-risk genotype infections occurred mainly in the chronic cervicitis and CIN groups but not in the SCC group $(P<0.01)$. This suggests that low-risk 
genotypes do not increase the risk of cervical cancer and are not crucial to its pathogenesis (Wang et al., 2010; Su et al., 2012). Furthermore, single-high-risk genotype HPV infections were the most common in the SCC group (63.82\%), occurring at a higher rate than multiple-high-risk infections $(18.60 \%)$ and multiple-high- and low-risk infections $(17.57 \%)(P<0.01)$. There was no significant difference between the proportions of multiple-high-risk infections and multiple-high- and low-risk infections $(P>0.05)$. All SCC specimens were positive for at least one high-risk genotype infection, which was not observed in the chronic cervicitis or CIN groups $(P<0.01)$. This confirms that highrisk HPV genotypes are a crucial factor in the pathogenesis of cervical cancer.

\section{Conflicts of interest}

The authors declare no conflict of interest.

\section{REFERENCES}

Bekkers RL, Massuger LF, BultenJ and Melchers WJ (2004). Epidemiological and clinical aspects of human papillomavirus detection in the prevention of cervical squamous cell cancer. Rev. Med. Virol. 14: 95-105. http://dx.doi.org/10.1002/ $\underline{\text { rmv.416 }}$

Bhatla N, Dar L, Rajkumar Patro A, Kumar P, et al. (2008). Human papillomavirus type distribution in women with and without cervical neoplasia in north India. Int. J. Gynecol. Pathol. 27: 426-430. http://dx.doi.org/10.1097/PGP.0b013e31816085ba

Clifford GM, Gallus S, Herrero R, Muñoz N, et al. (2005). World wide distribution of human papillomavirus types in cytologically normal women in the International Agency for Research on squamous cell cancer HPV prevalence surveys: a pooled analysis. Lancet 366: 991-998. http://dx.doi.org/10.1016/S0140-6736(05)67069-9

Cuschieri KS, Cubie HA and Whitley MW (2004). Multiple high risk HPV infections are common in cervical neoplasia and young women in a cervical screening population. J. Clin. Pathol. 57: 68-72. http://dx.doi.org/10.1136/jcp.57.1.68

de Sanjosé S, Diaz M, Castellsagu X, Clifford G, et al. (2007). Worldwide prevalence and genotype distribution of cervical human papillomavirus DNA in women with normal cytology: a meta-analysis. Lancet Infect. Dis. 7: 453-459. http://dx.doi. org/10.1016/S1473-3099(07)70158-5

Grce M, Matovina M, Milutin-Gasperov N and Sabol I (2010). Advances in cervical squamous cell cancer control and future perspectives. Coll. Antropol. 34: 731-736.

Li H, Yuan F and Zhang LM (2012). Human papillomavirus (HPV) infection in clinical status and its relationship with cervical cancer. Zhongguo Linchuang Yanjiu 25: 373-374.

Li J, Wang YY, Wang P, Sun L, et al. (2013). 2387 cases of HPV infection in Xi 'an of Shaanxi province and subtype distribution analysis. Xiandai Zhongliu Yixue 21: 2838-2842.

Li J, Wang YX, Tian XF, Wang P, et al. (2014). Analysis of human papillomavirus infection and typing in Shanxi province. Zhonghua Yu Fang Yi Xue Za Zhi 48: 192-196.

Li WX, Chen PP and Lv HX (2009). Human papillomavirus (HPV) infection distribution and its relationship with cervical lesions. Zhongguo Weisheng Jianyan Zazhi 19: 2590-2592.

Li ZF, Xie ZF, Zhang S, Wang G, et al. (2011). Analysis of cervical disease subtypes of HPV infection in Suzhou. Jiangsu Yufang Yixue 22: 7-9.

Lowy DR and Schiller JT (2006). Prophylactic human papillomavirus vaccines. J. Clin. Invest. 116: 1167-1173. http://dx.doi. org/10.1172/JCl28607

Muhanmode YLK and Liu KJ (2010). HPV in cervicitis, cervical cancer lesion before, detection and significance of cervical cancer. Xinjiang Yike Daxue Xuebao 33: 160-162.

Rao HY, Liu WG and Wang Y (2012). HPV genotypes in cervical cancer and precancerous lesions in the distribution. Zhongguo Weisheng Jianyan Zazhi 22: 121-122.

Sandri MT, Riggio D, Salvatici M, Passerini R, et al. (2009). Typing of human papillomavirus in women with cervical lesions: prevalence and distribution of different genotypes. J. Med. Virol. 81: 271-277. http://dx.doi.org/10.1002/jmv.21382

Spinillo A, Dal Bello B, Gardella B, Roccio M, et al. (2009). Multiple human papillomavirus infection and high grade cervical intraepithelial neoplasia among women with cytological diagnosis of atypical squamous cells of undetermined significance or low grade squamous intraepithelial lesions. Gynecol. Oncol. 113: 115-119. http://dx.doi.org/10.1016/j. ygyno.2008.12.037 
Stanley MA, Pett MR and Coleman N (2007). HPV: from infection to squamous cell cancer. Biochem. Soc. Trans. 35: 14561460. http://dx.doi.org/10.1042/BST0351456

Steben M and Duarte-Franco E (2007). Human papillomavirus infection: epidemiology and pathophysiology. Gynecol. Oncol. 107: S2-S5. http://dx.doi.org/10.1016/j.ygyno.2007.07.067

Su SH, Chen XQ and Yu J (2012). Human papillomavirus (HPV) infection of multiple different types of relationship with the degree of cervical lesions. Zhonghua Yiyuanganranxue Zazhi 22: 5292-5294.

Tao PP, Bian ML, Ou H, Li Y, et al. (2006). Diversion of hybrid chip technology in the study of the application of the nipple virus detection. Zhonghua Fuchanke Zazhi 41: 43-47.

Tovar JM, Bazaldua OV, Vargas L and Reile E (2008). Human papillomavirus, cervical squamous cell cancer, and the vaccines. Postgrad. Med. 120: 79-84. http://dx.doi.org/10.3810/pgm.2008.07.1794

Trottier H, Mahmud S, Costa MC, Sobrinho JP, et al. (2006). Human papillomavirus infections with multiple types and risk of cervical neoplasia. Cancer Epidemiol. Biomarkers Prev. 15: 1274-1280. http://dx.doi.org/10.1158/1055-9965.EPI-06-0129

Wang GY, Liu CJ, Peng Q, Xu X, et al. (2010). Human papillomavirus (HPV) infection and to explore the relationship between cervical lesions. Zhonghua Yi YuanGan RanXueZaZhi 20: 1628-1630.

Wu Y, Chen Y, Li L, Yu G, et al. (2006). Associations of high-risk HPV types and viral load with cervical squamous cell cancer in China. J. Clin. Virol. 35: 264-269. http://dx.doi.org/10.1016/j.jcv.2005.07.011

Yang H, Shi WJ and Chen W (2010). Cervical cancer and precancerous lesion of HPV infection and genotype distribution. China Healthy Birth Genet. J. 18: 42-43.

Zhang T, Lou XL, Li Y, Lu C, et al. (2009). Cervical diseases HPV detection and analysis of mixed infection. Prog. Obstet. Gynecol. 18: 11-13.

Zhao $\mathrm{AH}$ and Zhang $\mathrm{HH}$ (2009). Human papilloma virus infection and cervical cancer and precancerous lesions in the related research. Ningxia YixueZazhi 31: 684-686. 